\title{
Aplasia cutis congenita in surviving co-twin after propylthiouracil exposure in utero
}

\author{
Ruth M. Löllgen ${ }^{1}$, Anne-Marie Calza ${ }^{2}$, Valérie M. \\ Schwitzgebel $^{3}$ and Riccardo E. Pfister ${ }^{1, *}$ \\ ${ }^{1}$ Department of Neonatology, University Hospital of \\ Geneva, 1211 Geneva, Switzerland \\ ${ }^{2}$ Department of Pediatric Dermatology, University \\ Hospital of Geneva, 1211 Geneva, Switzerland \\ ${ }^{3}$ Department of Pediatric Endocrinology and Diabetology, \\ University Hospital of Geneva, 1211 Geneva, Switzerland
}

\begin{abstract}
Aim: Aplasia cutis congenita (ACC) has been observed after fetal exposure to the antithyroid drug methimazole (MMI), but not reported after propylthiouracil (PTU), the current antithyroid drug of choice during pregnancy. This occurrence has implications for patient information and causal research.

Case report: We describe a surviving term co-twin to a mother with hyperthyroidism exposed to PTU from conception to 34 weeks of gestation presenting with ACC at birth.

Discussion: The association between PTU exposure and ACC is clinically relevant and allows speculation on the etiology. A similar mechanism to the classical MMI-induced ACC is postulated, unless a vascular etiology suggested by a vanishing twin or maternal hyperthyroidism itself is causal. Coincidence of PTU exposure and ACC seems unlikely.

Conclusion: ACC in a newborn after PTU exposure during pregnancy hitherto observed only after MMI strongly encourages further reports of similar cases that may remain clinically underdiagnosed or unreported. Such confirmation could have significant implications for maternal treatment of hyperthyroidism, common in women of childbearing age.
\end{abstract}

Keywords: antithyroid drugs; aplasia cutis congenital; methimazole; propylthiouracil; vanishing twin.

\section{Introduction}

Aplasia cutis congenita (ACC) is part of a heterogeneous group of disorders characterized by the absence of epidermis in a localized or widespread area of skin at birth, sharply demarcated and non-inflammatory in appearance (1). This

\footnotetext{
*Corresponding author: Dr. R.E. Pfister, Neonatology Unit, University Children's Hospital, 6, Rue Willy-Donzé, 1211 Geneva, Switzerland Phone: +41-22-3824351, Fax: +41-22-3824365,

E-mail: riccardo.pfister@hcuge.ch
}

anomaly occurs spontaneously in 1 in 2000 births (2). Solitary defects on the scalp are most common (70\%) but multiple lesions may occur on the face, trunk or limbs, sometimes symmetrically. The defects may vary in size (between 0.5 and $10 \mathrm{~cm}^{2}$ ) and shape (circular, oval, linear, star-shaped configuration). Defects that form early in pregnancy may heal completely up to delivery and appear as atrophic, membranous or parchment-like scars with associated alopecia when on the scalp. Less mature defects appear as superficial erosions or deep ulcerations. The depth may involve the epidermis and upper or deep dermis, the subcutaneous tissue and rarely the periostium, the skull, the dura or the meninges. ACC is mostly a benign isolated defect but can be associated with other anomalies or malformation syndromes. A classification into nine groups has been proposed by Frieden (1).

Small and large defects on areas other than the scalp will heal spontaneously over several weeks with conservative skin care using antiseptic ointment by gradual epithelialization and formation of a hairless, atrophic scar. Even small underlying bone defects usually close spontaneously during the first year of life. By contrast, large or multiple scalp defects usually need excision and primary closure, if feasible. Full-thickness defects of the scalp, skull and dura are associated with more significant morbidity and mortality $(20 \%)$ and may require surgical intervention (3).

There is currently no unifying theory explaining the genesis of ACC. Mechanisms causing ACC comprise genetic factors, teratogens, vasculature accidents, and trauma or early rupture of the amniotic membranes and amniotic bands.

\section{Case report}

We report on a term newborn whose mother (gravida=2, parity=2) had clinical signs of hyperthyroidism at a preconception check, with goiter, tachycardia and moderate exophthalmia. Thyroid function tests (TSH $0.01 \mathrm{mU} / \mathrm{L}, \mathrm{fT} 3$ $22 \mathrm{pmol} / \mathrm{L}$ and fT4 $54 \mathrm{pmol} / \mathrm{L}$ ) and elevated anti-TSH receptor antibodies measured later in pregnancy (10.8 IU/L) confirmed the diagnosis of Grave's disease. Propylthiouracil (PTU) treatment was initiated 2 months before conception at $50 \mathrm{mg}$ PTU/day combined with a beta-blocker (propranolol). During pregnancy, fT3 and fT4 normalized, whereas TSH remained suppressed and anti-TSH receptor antibodies elevated. Antithyroid treatment was stopped at 34 weeks of gestation, as the mother was euthyroid.

This monochorionic bi-amniotic pregnancy remained clinically uneventful until routine ultrasound at 14 weeks revealed a vanishing twin syndrome. However, in the absence of visible congenital anomaly of the deceased twin or 
placental infarction at birth, no causal diagnosis was possible. Ultrasound follow-up of the surviving twin did not reveal fetal goiter and carefully monitored fetal heart rate remained in the normal range. No further complications occurred during pregnancy; in particular, no signs of fetal hypothyroidism were observed. Serology for toxoplasmosis, rubella, CMV, listeria, hepatitis B and C and HIV was negative and immunity for varicella was present.

The newborn girl (3150 g, $51 \mathrm{~cm}$ long, head circumference $33 \mathrm{~cm}$ ) had excellent adaptation at birth (APGAR 9/10/10). She had no clinical signs of neonatal hyperthyroidism such as tachycardia, hyperthermia or goiter, but anti-TSH receptor antibodies were elevated (10 UI/L; normal range $<1 \mathrm{IU} / \mathrm{L}$ ) and TSH was suppressed $(0.07 \mathrm{mU} / \mathrm{L})$. As thyroid function tests worsened ( $\mathrm{TSH}<0.01 \mathrm{mU} / \mathrm{L})$, antithyroid treatment with PTU was initiated on day 12 of life $(3 \times 5 \mathrm{mg} /$ day initially, $3 \times 2.5 \mathrm{mg} /$ day after improvement of thyroid function tests), and was stopped at 30 days of life. Thereafter, thyroid function tests remained within the normal range.

Skin lesions typical for ACC were evident at birth, and the association with vanishing twin syndrome allowed classification into group 5 (Table 1). Lesions were symmetrical. The largest lesion on the right flank was round in shape and superficially eroded $(1 \mathrm{~cm} \times 0.5 \mathrm{~cm})$ and surrounded by a scar of $7 \mathrm{~cm} \times 2.5 \mathrm{~cm}$ vanishing into star-shaped, parchment-like skin on the left flank that was $3 \mathrm{~cm}$ long (Figure 1). Four additional discrete linear scars were noted on each inner infrapatellar region and each thigh. No scalp lesions or associated anomalies were present. Conservative treatment with antiseptics led to rapid and complete scarring.

\section{Discussion}

Maternal hyperthyroidism may pose a risk to the fetus. To minimize the risk of complications such as fetal cardiac failure, craniostenosis, intrauterine growth restriction, prematurity, fetal hydrops and intrauterine death (4), pregnancy should be delayed until hyperthyroidism is under control. However, antithyroid treatment during pregnancy is recommended for newly developing hyperthyroidism, as well as recurrences, in the expecting mother (2). Lowest possible doses of antithyroid drugs are recommended to keep fT4 levels within the

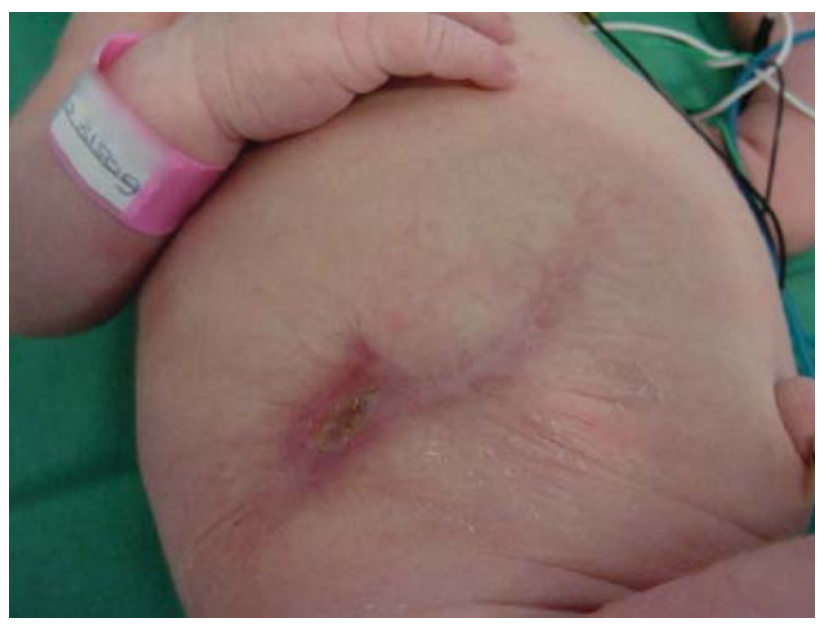

Figure 1 Aplasia cutis congenita of the right flank on day 5. Note the linear configuration with the adjacent wrinkly skin, a sign of a much larger fetal lesion.

high normal reference range and at the same time prevent placental drug-crossing causing fetal hypothyroidism, goiter and abnormal brain development (5).

In $1 \%-5 \%$ of cases, Grave's disease during pregnancy will lead to fetal and neonatal hyperthyroidism through placental crossing of thyroid-stimulating antibodies (thyrotropin-receptor antibodies). The risk of neonatal hyperthyroidism may be assessed by maternal thyrotropin-receptor antibodies at the beginning of the third trimester (6). Fetal signs of hyperthyroidism are poor growth and tachycardia, whereas neonates may present with tachycardia in addition to hyperactivity, irritability, hyperthermia and weakness.

To date, PTU is considered the antithyroid drug of choice during pregnancy compared to methimazole (MMI), which has considerable adverse fetal effects. Intrauterine exposure to MMI has been associated with congenital anomalies, in particular aplasia cutis congenita $(2,7)$, as well a syndrome called MMI embryopathy characterized by facial anomalies, choanal or esophageal atresia, tracheo-esophageal fistula, growth restriction, developmental anomalies and isolated imperforate anus (8). The incidence of spontaneous or inherited ACC is approximately 1:2000 births (2) and is limited in

Table 1 Classification of ACC according to Frieden (1).

\begin{tabular}{ll}
\hline Group & Description \\
\hline 1 & Solitary scalp ACC (86\%), with an autosomal dominant inheritance pattern or spontaneous \\
2 & Scalp involvement with limb anomalies; Adam Oliver syndrome is a subtype with distal limb reduction anomalies \\
3 & Scalp ACC with epidermal and sebaceous nevi involving the scalp \\
4 & ACC often with a hair collar overlying a deeper embryologic malformation such as meningomyelocele, gastroschisis, \\
& omphalocele, etc. \\
6 & Extensive truncal and limb ACC in a linear or star-shaped configuration, associated with fetus papyraceous or placental infarction \\
7 & ACC with simplex, junctional or dystrophic types of epidermolysis bullosa (EB) \\
8 & Localized ACC to the limbs with no EB \\
9 & ACC due to teratogens such as methimazole or carbimazole, misoprostol or valproic acid, or to intrauterine infection with \\
& ACC linked to malformation syndromes
\end{tabular}


the vast majority of cases to the scalp (Table 1); the ACC rate after maternal MMI treatment, however, remains unknown.

PTU and MMI cross the placenta similarly $(9,10)$. However, the theoretical risk for PTU is lower owing to higher levels of drug binding to serum proteins (11). Both drugs are classified as category D by the Food and Drug Administration because of placental transfer and the potential for fetal hypothyroidism (12). A similar incidence of major congenital anomalies has been reported after PTU (3\%) and MMI (2.7\%) exposure in utero (13). Several studies failed to show an increase in major congenital malformations in infants of hyperthyroid mothers treated with PTU (14-16). A study including 65 infants born to mothers with Grave's disease treated with either PTU or MMI again found no excess congenital anomalies (17). No case of ACC has been observed after maternal PTU treatment so far, but associations with other congenital anomalies have been reported, including congenital hip dislocation (18), undescended testes and muscular hypotony (19), hypospadias and aortic atresia (7), choanal atresia (20), ventricular septum defect, pulmonary stenosis, patent ductus arteriosus (13) and syndactily after combined PTU and ${ }^{131}$ I therapy (21). A cause of effect remains questionable in these mostly frequent anomalies.

The ACC reported here may be classified as group 5 (Table 1) because of its association with a vanishing twin. However, in utero exposure to PTU is of concern and teratogenic origin (Table 1; group 8) may also be postulated.

According to the current pathogenic hypothesis, symmetrical ACC is caused by a vascular disruption, either ischemic or thrombotic, affecting the dermo-epidermal development. Intra-uterine death of a twin may cause release of thrombotic material, promoting ACC in the surviving twin in the case of vascular shunts. This is usually associated with extensive placental infarctions, disseminated intravascular coagulation and other direct and usually severe injuries to the surviving twin, which we failed to find in our index case $(22,23)$.

So far, only MMI exposure has been incriminated as a cause of ACC (group 8), although MMI and PTU have the same pharmacological effect on the thyroid. Both inhibit thyroid hormone synthesis by acting on thyroid-peroxidase-mediated iodination of tyrosine residues in thyroglobulin, an important step in thyroxine and triiodothyronine synthesis (12). MMI has faster onset of action and prolonged activity, and readily crosses the placenta in the absence of binding proteins, and thus theoretically presents a higher risk to the fetus. Our finding suggests a similar teratogenic effect of PTU, possibly of vascular origin, unless the underlying maternal thyroid disease itself caused ACC.

Indeed, it has been suggested that hyperthyroidism per se is teratogenic $(2,24)$, but it has not been directly associated with ACC. Other anamnestic risk factors for ACC, including genetic components, other teratogens, vascular accidents, trauma or early rupture of the membranes with possible amniotic bands, were absent. Furthermore, our case may have resulted from the coincidence of spontaneously occurring ACC and PTU exposure in utero. Coincidence of ACC (1:2000 births) and PTU treatment (1:100-1:2000 pregnancies) (25) has a theoretical incidence of 1 in 2-4 million births, and would thus be an extremely rare event. For Switzerland alone, such a coincidence would present only every $30-60$ years. Thus, it is unlikely that this is the cause of our case, which is further supported by the fact that ACC group 5 is not a common presentation. However, coincidence with a vanishing twin, despite remaining rare, is more likely (26).

In conclusion, the reported new association between ACC and fetal exposure to PTU remains suspect for a druginduced pathology. To date, ACC has been reported only after exposure to MMI. Coincidence seems unlikely. Our finding allows speculation on a vascular etiology of ACC after maternal antithyroid drug exposure. The teratogenic risk of PTU is considered low at present, but its safety may be challenged by similar reports on ACC after PTU exposure in utero. As the clinical appearance of ACC can vary greatly and may remain undiagnosed when minimal or healed (wrinkly or parchment-like skin), neonatologists and dermatologists should complete a case history regarding drug intake during pregnancy and search for lesions in babies of mothers on PTU treatment. Until more cases have been reported or new strategies for maternal antithyroid treatment during pregnancy are developed, PTU will most likely remain the drug of choice, but a more systematic investigation of similar cases is warranted. If our report is confirmed, it could have significant implications for maternal treatment of hyperthyroidism, a common pathology in women of childbearing age.

\section{References}

1. Frieden I. Aplasia cutis congenita: a clinical review and proposal for classification. J Am Acad Dermatol 1986;14:646-60.

2. Mandel SJ, Brent GA, Larsen PR. Review of antithyroid drug use during pregnancy and report of a case of aplasia cutis. Thyroid 1994;4:129-33.

3. Martinez-Regueira S, Vazquez-Lopez ME, Somoza-Rubio C, Morales-Redondo R, Gonzalez-Gay MA. Aplasia cutis congenita in a defined population from northwest Spain. Pediatr Dermatol 2006;23:528-32.

4. Weetman A. Graves' disease. N Engl J Med 2000;343:1236-48.

5. Clark SM, Saade GR, Snodgrass WR, Hankins GD. Pharmacokinetics and pharmacotherapy of thionamides in pregnancy. Ther Drug Monit 2006;28:477-83.

6. Davies TF, Roti E, Braverman LE, DeGroot LJ. Thyroid controversy stimulating antibodies. J Clin Endocrinol Metab 1998;83: 3777-85.

7. Mujtaba Q, Burrow GN. Treatment of hyperthyroidism in pregnancy with propylthiouracil and methimazole. Obstet Gynecol 1975;46:282-6.

8. Di Gianantonio E, Schaefer C, Mastroiacovo PP, Cournot MP, Benedicenti F, et al. Adverse effects of prenatal methimazole exposure. Teratology 2001;64:262-6.

9. Mortimer RH, Cannell GR, Addison RS, Johnson LP, Roberts MS, et al. Methimazole and propylthiouracil equally cross the perfused human term placental lobule. J Clin Endocrinol Metab 1997;82:3099-102.

10. Gardner D, Cruikshank DP, Hays PM, Cooper DS. Pharmacology of propylthiouracil (PTU) in pregnant hyperthyroid women: correlation of maternal PTU concentrations with cord serum thyroid function tests. J Clin Endocrinol Metab 1986;62:217-20. 
11. Momotani N, Noh JY, Ishikawa N, Ito K. Effects of propylthiouracil and methimazole on foetal thyroid status in mothers with Graves' hyperthyroidism. J Clin Endocrinol Metab 1997; 82:3633-6.

12. Cooper DS. Antithyroid drugs. N Engl J Med 2005;352:905-17.

13. Wing DA, Millar LK, Koonings PP, Montoro MN, Mestman JH. A comparison of propylthiouracil versus methimazole in the treatment of hyperthyroidism in pregnancy. Am J Obstet Gynecol 1994;170:90-5.

14. Holt WA, Talbert L, Thomas CG Jr, Rankin P. Hyperthyroidism during pregnancy. Obstet Gynecol 1970;36:779-85.

15. Goluboff LG, Sisson JC, Hamburger JI. Hyperthyroidism associated with pregnancy. Obstet Gynecol 1974;44:107-16.

16. Ghaneim A, Atkins P. Management of thyrotoxicosis in pregnancy. Int J Clin Pract 1998;52:36-8.

17. Momotani N, Ito K. Treatment of pregnant patients with Basedow's disease. Exp Clin Endocrinol 1991;97:268-74.

18. Greenman GW, Gabrielson MO, Howard-Flanders J, Wessel MA. Thyroid dysfunction in pregnancy. N Engl J Med 1962;267: 426-31.
19. Herbst AL SH. Hyperthyroidism during pregnancy. N Engl J Med 1965;273:627-33.

20. Cheron RG, Kaplan MM, Larsen PR, Selenkow HA, Crigler JF Jr. Neonatal thyroid function after propylthiouracil therapy for maternal Graves' disease. N Engl J Med 1981;304:525-8.

21. Hollingsworth DR, Austin E. Thyroxine derivatives in amniotic fluids. J Pediatr 1971;79:923-9.

22. Cambiaghi S, Schiera A, Tasin L, Gelmetto C. Aplasia cutis congenita in surviving co-twins: 4 unrelated cases. Pediatr Dermatol 2001;18:511-5.

23. Leauté-Labreze C, Depaire-Duclos F, Sarlangue J, Fontan D, Sandler B, et al. Congenital cutaneous defects as complications in surviving co-twins. Arch Dermatol 1998;134:1121-4.

24. Momotani N, Ito K, Hamada N, Ban Y, Nishikawa Y, et al. Maternal hyperthyroidism and congenital malformations in the offspring. Clin Endocrinol (Oxf) 1984;20:695-700.

25. Mandel SJ, Cooper DS. The use of antithyroid drugs in pregnancy and lactation. J Clin Endocrinol Metab 2001;86:2354-9.

26. Landy HJ, Keith LG. The vanishing twin: a review. Hum Reprod Update 1998;4:177-83. 